Conclusion Sequential OAV therapy following treatment failure with PEG-IFN $\alpha$ is associated with greater reductions of $\mathrm{HBsAg}$ than PEG-IFN $\alpha$ alone or OAV monotherapy. This suggests PEG-IFN $\alpha$ may prime the immune response, even in the context of treatment failure, leading to better responses with sequential OAV therapy. Further studies are needed to confirm this finding and determine whether a similar priming effect is observed with shorter courses of PEG-IFN $\alpha$ in line with current PEG-IFN $\alpha$ stopping rules.

Disclosure of Interest None Declared.

\section{PWE-144 FRAX SCORE IN THE ASSESSMENT OF BONE MINERAL DENSITY CHANGES IN TENOFOVIR TREATED CHRONIC HEPATITIS B PATIENTS}

doi:10.1136/gutjnl-2013-304907.432

1."U S Gill, ${ }^{2} \mathrm{~A}$ Zissimopoulos, ${ }^{2} \mathrm{~S}$ Al-shamma, ${ }^{2} \mathrm{~K}$ Burke, ${ }^{3} \mathrm{M}$ McPhail, ${ }^{3} \mathrm{D}$ Barr, ${ }^{4} \mathrm{~V}$ Ross, ${ }^{2} \mathrm{Y}$ Kallis, ${ }^{2} \mathrm{P}$ Kooner, ${ }^{2} \mathrm{R}$ Marley, ${ }^{1} \mathrm{G}$ R Foster, ${ }^{1} \mathrm{P} T$ Kennedy. ${ }^{1}$ The Liver Unit, Blizard Institute, Barts and The London SMD, QMUL; ${ }^{2}$ Hepatology, Barts Health NHS Trust; ${ }^{3}$ Department of Medicine, Imperial College; ${ }^{4}$ Pharmacy, Barts Health NHS Trust, London, UK

Introduction Tenofovir Disoproxil Fumarate (TDF) is an established oral antiviral (OAV) in the treatment of Chronic Hepatitis B (CHB). Bone Mineral Density (BMD) loss has been described in TDF treated HIV patients, but limited data exist in $\mathrm{CHB}$. We have used DEXA scanning to determine BMD changes in TDF treated patients and have reported the possibility of BMD loss. DEXA scanning, however, is costly and requires longitudinal follow-up. We assessed the value of the FRAX ${ }^{\circledR}$ score and of bone biochemistry to evaluate their utility in TDF treated patients.

Methods The FRAX score is a WHO web-based tool, used to calculate 10-year fracture risk and the need for lifestyle modification, DEXA scanning or preventative treatment. CHB patients treated with TDF for a minimum of 12-months and a control group not exposed to TDF were studied. 122 TDF exposed patients (male $=89$ ), median age 45 (range $=26-64$ ) and 48 patients (male $=31$ ), median age 36 (range $=20-62$ ) not exposed to TDF were DEXA scanned and included in the study. We calculated FRAX scores and recorded bone biochemical markers, comprising serum Alkaline Phosphatase (sALP), Calcium (sCa) and Phosphate (sPO).

Results TDF treated patients had lower hip T-scores compared to controls $(p=0.02)$. On univariate analysis factors associated with a hip T-score $<1$ included older age, lower BMI, smoking and TDF exposure $(p=<0.05)$. On multivariate analysis the same factors were associated with a hip T-score $<1$, but TDF lost significance. For the development of a major osteoporotic fracture the pre-DEXA FRAX score was $4.77 \%$ compared to $4.33 \%$ (post-DEXA FRAX) $(p=0.9)$ and for a hip fracture this was $0.54 \%$ (pre-DEXA FRAX) and $0.77 \%$ (post-DEXA FRAX) $(p=0.5)$. The pre-DEXA FRAX score was a significant predictor of the post-DEXA FRAX treatment recommendation ( $p=<0.001)$. TDF therapy was associated with increased sALP after 12 -months, but this was not significant. No change was observed in pre-treatment sCa and sPO levels compared to those after 12 -months exposure ( $p=0.5 \& 0.09$ respectively).

Conclusion Our results demonstrate the FRAX score alone can accurately predict the risk of developing an osteoporotic fracture in TDF treated CHB patients. This potentially obviates the need for DEXA scanning and the associated costs. The relationship between sALP and TDF is noteworthy, but bone parameters are of limited use in predicting $\mathrm{BMD}$ changes. Although BMD loss in TDF treated $\mathrm{CHB}$ patients remains unproven, we demonstrate the use of the FRAX score may determine those at risk of osteoporotic fractures in $\mathrm{CHB}$.

Disclosure of Interest None Declared.

\section{PWE-145 RESTROSPECTIVE REVIEW OF HEPATITIS B DATABASE}

doi:10.1136/gutjnl-2013-304907.433
1."V Sagar, ${ }^{2} \mathrm{~N} N$ Than, ${ }^{3} \mathrm{~S}$ Naqvi, ${ }^{4} \mathrm{~S}$ Singhal. ${ }^{1}$ Medicine, Heart of UK NHS Foundation Trust; ${ }^{2}$ Gastroenterology; ${ }^{3}$ Medicine, University Hospitals Birmingham NHS Foundation Trust; ${ }^{4}$ Gastroenterology, Sandwell and West Birmingham Hospitals NHS Trust, Birmingham, UK

Introduction Chronic Hepatitis B Virus (HBV) affects 350 million people worldwide with potential serious consequences. The aim of this study was to measure adherence at Sandwell and West Birmingham Hospitals (SWBH) NHS Trust to recent guidance regarding HBV assessment and treatment.

Methods A retrospective review of the SWBH HBV database (2003-2012) was undertaken. On the basis of European Association for the Study of the Liver (EASL) guidance, attainment of the following outcomes (standard 100\%) was calculated: ALT measurement, liver ultrasound (US) examination, HBV DNA measurement, HIV testing, further liver assessment where indicated (using biopsy or Fibroscan) and use of antiviral therapy where indicated.

Results 322 patients with HBV were identified. Attainment of the EASL standards were as follows: ALT measurement 92\%, liver US examination $80 \%$, HBV DNA measurement $95 \%$, HIV testing $72 \%$, further liver assessment where indicated $82 \%$ and use of antiviral therapy where indicated $100 \%$.

Conclusion In general patients were managed according to EASL guidance. Liver US examination was not 100\% mainly because patients failed to attend their appointment. HIV testing was not $100 \%$ as routine testing in HBV patients was introduced only in 2008. Further liver assessment with biopsy was deferred in a number of cases after discussion between patient and physician; recent acquisition of a Fibroscan at SWBH should increase the proportion of appropriate patients undergoing further liver assessment. It is encouraging that all patients received antiviral therapy where indicated. It is hoped that data from this review and recent acquisition of a Fibroscan at SWBH Trust will promote improved adherence to guidelines.

Disclosure of Interest None Declared.

\section{REFERENCES}

1. AASLD practise guidelines 2009: Chronic Hepatitis B

2. EASL Clinical Practice Guidelines: management of chronic hepatitis B. European Association For The Study Of The Liver. J Hepatol. 2009 Feb; 50(2):227-42

\section{PWE-146 RELAXIN IS A RENAL VASODILATOR IN EXPERIMENTAL MODELS OF CIRRHOSIS AND A POTENTIAL NOVEL THERAPY FOR HEPATORENAL SYNDROME IN HUMANS}

doi:10.1136/gutjnl-2013-304907.434

1." V K Snowdon, 'A Pellicoro, 'P Ramachandran, ${ }^{2} \mathrm{~W}$ Mungall, ${ }^{3} \mathrm{M}$ Jansen, ${ }^{3} \mathrm{R}$ Lennen, ${ }^{1} \mathrm{R}$ Aucott, 'T Kendall, ' $\mathrm{J}$ Hughes, ' J P Iredale, ' J A Fallowfield. 'MRC/Centre for Inflammation Research, Queens Medical Research Institute, University of Edinburgh; ${ }^{2}$ Biomedical Research Resources, University of Edinburgh; ${ }^{~} E$ dinburgh Preclinical Imaging, BHF/ University Centre for Cardiovascular Science, University of Edinburgh, Edinburgh, UK

Introduction Hepatorenal syndrome (HRS) is a feared complication of cirrhosis with a high mortality rate and limited treatment options. The hallmark features of HRS are profound renal vasoconstriction, resulting in a functional renal failure but with normal kidney histology. The peptide hormone relaxin (RLN) mediates maternal haemodynamic adaptations to pregnancy, including increased renal blood flow (RBF) and glomerular filtration rate (GFR). We hypothesised that RLN could beneficially modulate RBF in cirrhosis and HRS.

Methods Cirrhosis, with reduced RBF, was induced in rats by 16 weeks of intraperitoneal (i.p.) carbon tetrachloride $\left(\mathrm{CCl}_{4}\right)$ and decompensated biliary cirrhosis by 3 weeks bile duct ligation (BDL). We measured the effect of acute intravenous (i.v.) and extended (72 hr) subcutaneous (s.c.) RLN on systemic haemodynamics, RBF, GFR and organ histology. Subgroups of rats were co-treated with the nitric oxide (NO) synthase inhibitor L-NAME. Blood oxygen 